MUZIKOLOSKI ZBORNIK - MUSICOLOGICAL ANNUAL XVII/1, LJUBLJANA 1981

UDK 78.01:930.1

\title{
ÜBERLEGUNGEN ZUR GESCHICHTE DER MUSIKHISTORIOGRAPHIE
}

\author{
Heinz Alfred B rockhaus (Berlin)
}

Die Musikgeschichte ist eine Fachdisziplin im komplexen Sinne des Wortes, sie faßt die Ergebnisse vieler Teildisziplinen zusammen, von der Musikethnologie über die Musikästhetik, Musiksoziologie, über die Instrumentenkunde bis zur Notations- und Quellenkunde, zur Stilkritik und Analyse, die sich in methodischen Grundsätzen unterscheiden, im historischen oder systematischen Konzept verschieden sind, ihren Gegenstand aber stets dem einen großen, totalen Sachverhalt entnehmen, der Geschichte der Musikkulturen. Sie wird von der $\mathrm{Mu}-$ sikgeschichte untersucht und umfaßt eine ihrem Wesen nach unendliche Totalität. So wie wir Geschichte heute begreifen, ist auch die Musikgeschichte nach ihren beiden Richtungen, nach Vergangenheit und Gegenwart hin offen, und es ist nicht möglich, bei der Analyse des Musikalischen, wie wir es im engeren Sinne verstehen, eine für alle Zeiten der Geschichte grundsätzlich gültige, konstant wirksame Bestimmung und Begrenzung der Fakten, der Voraussetzungen, Einflüsse, Kausalbeziehungen etc. zu definieren. Die Problematik des NichtEndlichen sondern Historischen gilt somit für die Zeit-Dimension und für die dialektische Determination musikalischer Sachverhalte im geschichtlichen Totalzusammenhang.

Schon diese knappen Hinweise veranschaulichen, daß Musikgeschichtsschreibung mehr verlangt, als nur Fakten zu erfassen und zu benennen. Die Aufgaben der Musikgeschichtsschreibung umschließen komplizierte philosophische Probleme, denn schon die Fragen »Was ist Musikgeschichte? « oder »Wie schreibt man eine Musikgeschichte? « sind philosophisch determinierte Fragen und verlangen entsprechend begründete Antworten, die meist viele weitere Fragen nach sich ziehen.

In der internationalen Praxis der Musikwissenschaft ist die Theorie der Musikgeschichte und Musikgeschichtsschreibung ein Arbeitsgebiet, das durchaus umstritten und von vielen Fragezeichen der Skepsis umgeben erscheint, das aber gerade deshalb der gezielten und konzentrier- 
ten Bearbeitung bedarf. Viele Ansätze sind vorhanden, sie verlangen nach Fortsetzung, Zusammenfassung und planmäßiger Untersuchung. Mit den Problemen der musikhistorischen Begriffsbildung haben sich Musikhistoriker vielerorts auseinandergesetzt, sei es mit dem Begriff Renaissance, dem Begriff Barock, der Klassik oder der Neuen Musik. Alle Musikgeschichtsschreibung erweist sich als angewandte Theorie, der Generationentheorie, der Jahrhundert- oder Zyklentheorie, der Evolutionstheorie und anderer, auch dann, wenn die theoretisch-konzeptionellen Probleme von den Autoren nicht ausdrücklich erörtert werden. Letztlich muß man wohl einen Schritt weiter gehen, um die volle Tragweite des Begriffs Theorie zu erfassen, wenn beispielsweise die Auffassung von der Abendland-Hegemonie, dem 'Eurozentrismus' oder von einer polyzentrischen Musikgeschichtsschreibung ins Auge gefaßt werden. Über alle diese Aspekte hinaus erweist sich die Geschichte der Musikhistoriographie als eine wesentliche Dimension einer jeden Theorie der Musikgeschichte.

Im Sinne einer Wissenschaftsdisziplin gibt es Musikgeschichte erst seit dem ausgehenden 18. und frühen 19. Jahrhundert. Als ein Nachdenken über musikgeschichtliche Sachverhalte, vielleicht auch im spekulativen Denken, hat es sie seit den Zeiten der altorientalischen Klassengesellschaft, in einzelnen Ansätzen sogar seit dem ausgehenden Neolithikum gegeben, als man begann, sich die Herkunft der Musik, die Erfinder der musikalischen Formen, der Instrumente oder Funktionen im religiösen Gewand vorzustellen. So ist die Mythologie der Frühgeschichte ein Ausgangspunkt musikgeschichtlichen Denkens, sie geht über in die Genealogie der orientalischen und antiken Klassengesellschaften und fließt von da aus weiter in die Vorstellungen des Hellenismus und der Patristik über die Herkunft und Funktion der Musik. Alles das, von uralten Sagen und Mythen über den musikschaffenden Göttervater Odin oder Zeus, über die Musen und Apoll, über die erfinderischen Kräfte des Orpheus oder Marsyas, bis hin zu den Anfängen geschichtlichen Denkens bei Herodot oder Thukydides, bei Plutarch, Livius oder Tacitus, in den Schriften der Kirchenväter von Augustinus über Boethius bis zu Cassiodor hatte noch keinen wissenschaftlichen Aussagecharakter, ist aber für die Forschungen der Gegenwart oft von großem Wert, weil es Quellenmaterial über die Vorstellungen der Menschen von der Herkunft des Musikalischen anbietet. Im gesamten Mittelalter ist musikwissenschaftliches Denken theologisch bestimmt, Aussagen zur Herkunft und zum Werdegang der Musik entweder rein pädagogisch konzipiert oder sie haben religiös-apologetische $\mathrm{Be}-$ deutung, tragen mit bei zum scholastischen Finalitätsdenken, das jedwede Erscheinungsform des Lebens, auch die Künste als Magd der Theologie und als Element zur Bestätigung der religiösen Dogmatik begreift. Erst in den kritisch konflikthaften Zeiten des 13. und 14. Jahrhunderts treten Autoren von Musiktraktaten auf, man denke an den Anonymus IV oder an Johannes de Grocheo, die sehr wohl kri- 
tische Positionen einnehmen, um eine Darstellung realer musikgeschichtlicher Sachverhalte bemüht sind, ohne allerdings eine Vorstellung von der Geschichte, von der Musikgeschichte und ihren inneren Gesetzmäßigkeiten zu haben. Bis zur Epochenwende vom Mittelalter zur Renaissance gibt es keine wissenschaftliche Musikgeschichtsauffassung, wohl aber sehr viel schriftliches Material, das den Vorstellungen jener Zeit über die Herkunft und Funktion der Musik Ausdruck verleiht. Trotz der sehr deutlichen Gegenwartsbezogenheit der nachfolgenden Epoche, der Renaissance, des Humanismus und der protestantischen Bewegungen, tritt in den Schriften der Renaissance-Theoretiker oder auch Luthers zum ersten Male Geschichtsbewußtsein, eine Geschichtsdimension in Erscheinung, mindestens in dem Sinne, daß man sich auf eine weiter zurückliegende Epoche, die Antike, bezieht und die unmittelbar vorausgehende, das Mittelalter, ablehnt. Nichtsdestotrotz ist beiden, dem Renaissance-Denken und der Selbstdarstellung des Reformators das gleiche Finalitätsdenken eigen, das schon in der mittelalterlichen und antiken Theorie hervortrat, die Ansicht, daß es Sinn der gesamten vorausgehenden Geschichte gewesen sei, die eigenen großen Zeiten, die allen anderen überlegen sind, heraufzuführen und möglich zu machen. Aus der Vereinigung des späten Renaissance-Denkens und des frühen Rationalismus erwachsen neue Anstöße zur Ausweitung des historischen Denkens. Sie äußern sich nun, bis gegen Ende der Aufklärung; in einer Verknüpfung chronistischer und enzyklopädischer Konzeptionen, die zunächst einmal bemüht sind, das vorhandene Wissen zu ordnen und zu systematisieren. So entstehen Schriften von Sethus Calvisius und Michael Praetorius, von Sebastien de Brossard, Pierre Bonnet und Pierre Bourdelot, kulminierend in den Beiträgen der Großen Enzyklopädie der französischen Aufklärer um 1750, die alle auf den Grundgedanken hinauslaufen, das gesammelte Wissen der Menschen um die Musik, also auch das historische Wissen aufzuschreiben und kritisch zu sichten. Das letztere war selbstverständlich zeitbedingt, also wiederum apologetisch, aber der chronistisch-enzyklopädische Aspekt dieses Arbeitens sicherte der Musikgeschichtsschreibung Materialkenntnisse, die heute äußerst wertvoll sind. Charakterististische Beispiele hierfür sind auch das Musik-lexikon von Johann Gottfried Walther, das Musikalische Wörterbuch von Brossard, das Tonkünstlerlexikon von Ernst Ludwig Gerber, die Allgemeine Theorie der Schönen Künste von Johann Georg Sulzer und noch das Handwörterbuch der Musik des Heinrich Christoph Koch, obwohl in diesem bereits der Umbruch in eine neue Zeit erkennbar wird. Aus dem Rationalismus, aus der Emanzipation des Bürgertums in der Feudalgesellschaft dieser Zeit erwächst die Aufklärung, beide Aufklärung und bürgerliche Emanzipation - beschleunigen den Prozeß der Nationenbildung. Alle drei Ansätze haben zur Folge, daß das geschichtliche Denken eine neue Qualität erreicht; so artikulieren sich innerhalb der rationalistisch aufklärerischen Denkweise die wesent- 
lichen Anfänge der wissenschaftlichen Musikgeschichtsschreibung. Sie beginnt als chronistische Arbeit, das heißt die Historiker sammeln und ordnen historisches Material in einer Chronographie, beschreiben das Material auch, erschließen jedoch die inneren Gesetzmäßigkeiten nach einem mechanisch in die Geschichte hineingedachten Denksystem, dem rationalistischen Fortschrittsbegriff. Diese rationalistische Vorstellung vom Fortschreiten in der Geschichte ist eine mechanische, weil sie generell annimmt, jeder Fortschritt sei als ein geradliniger Aufstieg vom Niederen zum Höheren anzusehen. Elemente dieser Vorstellung finden sich bei Wolfgang Caspar Printz, der 1690 seine »Historische Beschreibung der Edlen Sing- und Klingkunst « herausbringt, bei Pierre Bonnet-Bourdelot, der 1715 eine Abhandlung über die Geschichte der Musik und ihrer Affekte veröffentlicht, bei dem Italiener Giovanni Battista Martini, der in der 2. Hälfte des 18. Jahrhunderts bereits eine hervorragende dreibändige Storia della Musica erscheinen läßt, schließlich neben einigen anderen in den Musikgeschichten der beiden berühmten Engländer, der fünfbändigen Musikgeschichte von John Hawkins von 1776 und vor allem in der 1776 bis 1789 folgenden Musikgeschichte von Charles Burney. Das Wesen dieser fundamentalen musikgeschichtlichen Darstellungen beruht auf einer universalen Dimension, der Zusammenfassung des gesamten vorliegenden, bekannten Materials, erweitert durch selbständige Forschungsarbeiten in Archiven und Bibliotheken, Reisen in die verschiedenen Länder und Zentren der Musikkultur und die Erarbeitung erster Werturteile. So haben die ältesten Musikhistoriker im eigentlichen Sinne des. Wortes die erste Etappe der modernen Musikhistoriographie geprägt, an sie haben sich nahezu alle Musikhistoriker des frühen 19. Jahrhunderts angeschlossen.

Daß auch die zur damaligen Zeit einsetzende Musikkritik zur Herausbildung des historischen Verständnisses musikalischer Sachverhalte beigetragen habe, bestätigen vor allem die Arbeiten von Johann Mattheson, der in die Erörterungen seiner »Critica Musica«, des "Vollkommenen Capellmeisters" und der "Ehrenpforte " musikgeschichtliche Wertungen einbezog, die jedoch auch hier stets gegenwartsbedingt und äußerst rechthaberisch sind.

In einem vierten Bereich erwächst musikgeschichtliches Denken aus dem pädagogischen Ansatz. Im Kontext aufklärerischer Bestrebungen des 18. Jahrhunderts entstehen in vielen Ländern Europas Lehrbücher, auch auf dem Gebiete der Musik. Repräsentanten dieser Arbeitsrichtung sind Johann Joachim Quantz, Carl Philipp Emanuel Bach, Leopold Mozart und Johann Adam Hiller. ${ }^{1}$ Sie haben ihren Schulwerken, die an sich dem korrekten Spiel, der Interpretation, der Ausdrucksästhe-

1 Quantz, J. J., Versuch einer Anweisung die Flûte traversière zu spielen, 1752; Bach, C. Ph. E., Versuch über die wahre Art das Clavier zu spielen, 1753, 1762; Mozart, L., Versuch einer gründlichen Violinschule, 1756; Hiller, J. A., Anweisung zum musikalisch richtigen Gesange, 1774. 
tik, den einschlägigen Kompositionen zugewandt sind, auch historiche Hinweise zur Geschichte des Instruments (beziehungsweise des Gesangs) und seiner Musik beigefügt. So haben sie schon damals die Konzeption für frühe Ansätze der Gattungsgeschichten geschaffen. Ungeachtet so wichtiger Beiträge spielt gerade zu dieser Zeit die Musik, oder richtiger die Musikwissenschaft an den Universitäten entweder gar keine oder nur eine klägliche Rolle; wenn überhaupt, dann war sie in akustische Vorlesungen der Physiker eingeordnet, gelegentlich erschien sie noch bei den Mathematikern. Die Ausbildung in diesem Fach erschöpfte sich in 12-16 Lektionen, die man in vier bis sechs Wochen erledigen konnte. Es war der Leipziger Wissenschaftsorganisator und Jurist Lorenz Mizler, der Mitte des 18. Jahrhunderts nicht nur neue Ansätze für die Organisation der Musikwissenschaft verfolgte, sondern auch die Wiedererrichtung von musikalischen Lehrstühlen forderte und voranzutreiben versuchte. Er selbst veranstaltete nach langer Pause in Leipzig erstmals wieder Lehrveranstaltungen dieser Art und vertrat den Standpunkt, »daß die Vernunft, die über alles herrschet, auch in der Musik herrschen müsse «.2 Auch hier ist also rationalistisches Denken die Triebfeder für neue Ansätze der Musikwissenschaft. Verwirklicht wurden solche Ansätze im 17. Jahrhundert in England, wo die Universitäten Oxford und Cambridge Lehrstühle für Musik erhielten - allerdings im Sinne der älteren Tradition. Im 18. Jahrhundert spitzte sich die Diskussion um eine Wiedererrichtung der $\mathrm{Mu}-$ sikwissenschaft - anfangs durchaus im Sinne der alten ars musica - vor allem an den deutschen Universitäten $\mathrm{zu}$. Johann Mattheson forderte 1739 eine Professur für Musikwissenschaft, ein Fach, das allen anderen Universitätsdisžiplinen gleichgestellt sei. 1758 beklagte Johann Adlung, daß es an deutschen Universitäten keine Lehrstühle gäbe, wie sie England bereits besitze. ${ }^{3} 1772$ wird Johann Nikolaus Forkel Universitätsorganist in Göttingen, er veranstaltet bald darauf privatim Lehrveranstaltungen zur Melodie- und Harmonielehre. Forkel war es, der den Anregungen des Göttinger Historikerkreises, den Ideen Herders, des Rationalismus und der Aufklärung folgend eine erste Geschichte der Musik zu schreiben versuchte. Sie erschien 1788 und 1801 und ist an sich ohne Herders Philosophie und Geschichtsauffassung nicht zu begreifen. In den geschichtswissenschaftlichen und philosophischen Arbeiten Johann Gottfried Herders kündigt sich der Übergang zur Epoche eines neuen historischen Denkens an. Mit fundamentalen Schriften wie den »Ideen zur Philosophie der Geschichte der Menscheit«, den »Briefen zur Beförderung der Humanität« und anderen hat er auch der Musikgeschichtsschreibung die entscheidenden Postulate einer historisch realistischen Methode vermittelt. Diese sind für die Beurteilung seiner wissenschaftsgeschichtlichen Position entscheidend, auch dann,

2 Mizler, L., zitiert nach MGG IX, Sp. 388.

3 Adlung, J., zitiert nach MGG XIII, Sp. 1093 ff. 
wenn man gegen die Parallelisierung natürlicher und gesellschaftlicher Fortschrittsgesetze den Einwand erheben mag, sie sei noch direkt aus den Normen des rationalistischen Denkens abgeleitet. Herders Idee jedoch, die Geschichte als eine fortschreitende Entwicklung zur Humanität zu begreifen, zeigt, daß auch die mechanistischen Auffassungen des Rationalismus den Zugang zu einer so programmatischen These ermöglichten. Forkels "Allgemeine Geschichte der Musik" war eine erste musikalische Universalgeschichte in Deutschland, sie blieb zwar Fragment, zeigte sich aber in ihrem historiographischen Konzept als Versuch, die musikgeschichtliche Darstellung mit der Kulturgeschichte und der allgemeinen Geschichte insgesamt zu verknüpfen.

Neben Forkel, der in Göttingen wirkte, gab es bereits ab 1826 eine außerordentliche Professor für Musik an der Universität Bonn, die jedoch nicht $\mathrm{zu}$ einer kontinuierlichen Entwicklung führen konnte. Der tatsächlich effektive Ansatz für die Herausbildung der Musikwissenschaft, der Musikgeschichte als Universitätsdisziplin im modernen Sinne des Wortes, wurde an der Berliner Universität geleistet, und dafür gab es eine Reihe von Gründen und unterschiedliche Voraussetzungen mit zum Teil problematischen Konsequenzen.

Wichtig ist jedenfalls, daß sich die Musikwissenschaft der Berliner Universität um 1829/1830 nicht aus einem völligen Neuansatz entwickelte, sozusagen aus einem Vakuum, sondern eher als die Kulmination eines weiträumigen Prozesses, der sich in besonders intensiven Anstößen und Vorbildern im Zeitalter der Aufklärung und der bürgerlichen Nationenibildung vollzog.

Die gesellschaftliche Situation in Preußen, in Berlin insbesondere, war eine politisch konservative, in der sich neue gesellschaftliche Ideen nur unter Mühen entfalten konnten. Während der Regierungszeit Friedrich Wilhelm III. (1797 bis 1840) setzten sich aber auch die einer großen Vergangenheit zugewandten Denkrichtungen fort, wie sie bereits vor der Jahrhundertwende dominiert hatten. Kennzeichnend für die zweite Hälfte des 18. Jahrhunderts in Berlin war, daß die großen Repräsentanten der zweiten Berliner Liederschule, Johann Abraham Peter Schulz und Friedrich Reichardt, aber auch Karl Friedrich Zelter während der nachfolgenden Jahre, oft gegen höfisch feudale Despotie ankämpfen mußten. Gerade diese Komponisten waren der Aufklärung, den Ideen der französischen Revolution, den Anschauungen des Freimaurertums jener Zeit zugewandt. Typisch für Schulz ist, daß er nicht nur als ein hervoragender Repräsentant demokratisch künstlerischer Bestrebungen anzusehen ist, man denke an seine "Lieder im Volkston", sondern auch als der konsequente Verfechter der Bauernbefreiung und weitgefächerter Reformen des Bildungs-, vor allem des musikalischen Bildungswesens. Unter dem Druck der höfisch konservativen Partei um die Prinzessin Amalie in Berlin mußte er 1787 ins Exil nach Kopenhagen gehen. Dort erarbeitete er weitere Materialien zu einer Schulreform; dazu gehört die im Jahre 1790 abge- 
faßte Konzeption »Gedanken über den Einfluß der Musik auf die Bildung eines Volkes...»

Trotz der schwierigen Position in Berlin unterhielt Johann Abraham Schulz enge Kontakte zu jenen Kreisen, die sich in Preußen um Bildungsreformen und eine Entfaltung des Musiklebens bemühten. Seine Ideen hat im wesentlichen Karl Friedrich Zelter fortgesetzt und weiterentwickelt, den man mit guten Recht den Vater der preußischen Musikkultur in der ersten Hälfte des 19. Jahrhunderts nennen darf. Er war überzeugter Anhänger der französischen Revolutionsideen, nach der Besetzung Berlins durch die Franzosen wurde er von den Bürgern in das Comité administratif gewählt, er war es, der durch eine Reihe von Memoranden immer wieder zur Entfaltung des Musiklebens, zur Einrichtung musikalischer Lehranstalten und zur Verbesserung des musikalischen Bildungssystems in umfassenden Sinne aufrief. Zelter war der erste Musiker, dem im Jahre 1830 der Doktorgrad ehrenhalber von der Universität Berlin verliehen wurde. Zu seinen persönlichen Schülern zählten nicht nur Musiker wie Felix Mendelssohn, Otto Nicolai, Carl Loewe und Eduand Grell, sondern auch der spätere Musikdirektor Bernhard Klein und der enste Professor für Musik an der Universität, Adolf Bernhard Marx. Zelter war es, der in seinen Denkschriften die Umwandlung der traditionellen handwerklich zünftig organisierten musikalischen Bildung in ein staatlich unterhaltenes System musikalischbildender Institutionen forderte, er propagierte die Einrichtung von Professuren für die allgemeine Musiklehre, wie sie dann auch verwirklicht wurde. Zelters Ansatz, an der Berliner Universität eine Professur für Musik einzurichten, war also in erster Linie kulturpolitisch und bildungspolitisch motiviert, und diesen Denkansatz brachte er auch in seine Gespräche mit Goethe, mit Mendelssohn, mit Wilhelm von Humboldt ein. So waren es die großen Traditionen der deutschen Musikkultur und die anderer Länder, deren Wiedererweckung und aktive Pflege er im Sinne seines Konzepts der Volksbildung propagierte. In diesen Bestrebungen traf er sich mit Felix Mendelssohn, der aus vielen Gesprächen im Kreise seiner Familie und im Freundeskreis der Eltern die Ideen des klassischen $\mathrm{Hu}-$ manismus, der Aufklärung, der Perfektibilität des Menschen und einer aktiven Entfaltung aller Schätze der Musikkultur mitbrachte. Zum Freundeskreis der Familie Mendelssohn gehörten Persönlichkeiten wie Schleiermacher, die Schlegels, Wilhelm von Humboldt, Hegel, August Boeckh, der Historiker Gustav Droysen, Heinrich Heine, Adolf Bernhard Marx, aber auch der Heidelberger Justus Thibaut, der über seine Vorstellungen berichtete, historische Konzerte einzuführen. Als Mendelssohn, der Sänger und Schauspieler Eduard Devrient und Karl Friedrich Zelter 1829 die Wiederaufführung der Matthäus-Passion Bachs durchsetzten, waren verschiedene Ansätze im Spiele: die Erhaltung großer Traditionen, ein großes Konzept der Volksbildung, die Perfektibilität jedes einzelnem Menschen, die Pflege, Aufführungs- 
praxis und auch Restauration alter Musik. Daß es sich hierbei um einen potentiellen Widerspruch handelte, war noch nicht erkennbar. Zunächst war es wahrscheinlich so, daß die Wideraufführung der Matthäus-Passion und das große, damit verbundene Erlebnis den letzten und entscheidenden Anstoß gab, an der Berliner. Universität eine Professur für Musik einzurichten. Lehrveranstaltungen zur allgemeinen Musiklehre, also eher musikalisch-praktische Bildungsmöglichkeiten, wurden in Berlin schon etliche Jahre zuvor angeboten, eingeordnet in nützliche und angenehme Übungen, vor allem der Tanz-, Reit- und Fechtkunst. Bernhard Klein veranstaltete solche musikalisch-praktischen Übungen bis zum Jahre 1832. In den Diskussionen der Jahre 1828/29 gab Karl Friedrich Zelter zunächst die Anregung, den jungen Felix Mendelssohn als ersten Professor der Musik an die Berliner Universität zu berufen. Dieser lehnte jedoch ab, und deshalb entschied man sich für Zelters Schüler Adolf Bernhard Marx, dessen Berufung 1829 ausgesprochen wurde. Analysiert man sein Wirken in den Jahren 1831 bis 1866, dann zeigt sich, in wie hohem Maß sein Denken Anstöße des rationalistischen Konzepts, romantische Ideen und Elemente der Hegelschen Dialektik in einer widerspruchsvollen Mischung zu vereinigen trachtete. Während seines gesamten Wirkens an der Berliner Universität las er Kompositionslehre und folgte damit ältesten Vorstellungen von der Funktion der Musikwissenschaft an der Universität, wie sie auch in England bereits verwirklicht waren und übrigens bis heute beibehalten wurden. Darüber hinaus trat Marx als Musikhistoriker hervor, der gegenüber mehr dilettantisch-autodidaktisch wirkenden Musikhistorikern wie Winterfeld oder Kiesewetter eine solide praktische musikalische Bildung besaß. Seine Biographien Beethovens und Glucks bezeugen eine Vermischungstendenz unterschiedlicher historiographisch konzeptioneller Ansätze, wie sie für eine Übergangsperiode typisch sind.

Überblickt man das Gesamtbild der musikgeschichtlichen Wirksamkeiten in der ersten Hälfte des 19. Jahrhunderts, so fällt die Vorrangstellung antiquarisch-archivalischer und philologischer Arbeitsweisen ins Auge. Was man zunächst einmal versuchte war, Schätze alter Musik so vollständig wie möglich $\mathrm{zu}$ sammeln, $\mathrm{zu}$ ordnen und einer textkritischen Sichtung zu unterziehen. Für den Musikhistoriker gab es zu dieser Zeit noch die Möglichkeit, das zu bearbeitende Material an einem bestimmten Ort seines Wirkens zu sammeln, und das waren dann auch quasi staatliche oder doch öffentliche Bibliotheken und Archive, oft genug aber repräsentative Privatsammlungen. Was sich zusammenfügte, waren Autographe, viele Abschriften, die die Sammler selbst anfertigten, theoretische Schriften, Bildmaterial und dann auch Instrumente. Relativ früh begannen einige Sammler mit dem Zusammentragen von Volksliedern. Dieser Ansatz, der in der Geschichte der Musikhistoriographie als die antiquarisch-archivalische Methode anzusehen ist, hat dann nicht nur die Quellensammlung und 
Quellenerschließung, sondern auch die Musikgeschichtsschreibung geprägt. Die Ansätze wurden in mehreren Ländern gleichzeitig vollzogen. So traten in der ersten Hälfte des 19. Jahrhunderts als Pioniere dieser Tätigkeit mehrere Musikwissenschaftler hervor, als einer der ersten offenkundig Joseph von Sonnleithner in Wien, der dort als einer der Gründer der Gesellschaft der Musikfreunde deren Archiv und Bibliothek aufbaute und mit viel Mühe und Fleiß Material zusammentrug, in einem bestimmten Falle die Bestände bereits bis zu Quellen des 9. Jahrhunderts ausdehnte, indem er ein neumiertes Antiphonar aus St. Gallen entdeckte. Sonnleithner war so einer der frühesten Repräsentanten der antiquarisch-archivalischen Methode. Ihm folgte, ungefähr eine Generation später, als wichtigster Repräsentant in Frankreich und Belgien François Joseph Fétis, der durch sein Wirken in Paris und Brüssel die Materialsammlung erstmals mit biographisch-bibliographischen Studien verknüpfte. Aufgrund seines Wirkens an Musiklehranstalten dachte Fétis in erster Linie musikalisch-praktisch und begann mit der Planung groß angelegter Editionen. Ebenfalls in Frankreich wirken zu dieser Zeit Edmond de Coussemaker und. François-Auguste Gevaert, in Berlin wenig später Robert Eitner, Franz Commer, ihnen folgend Siegfried Dehn, und in Wien schließlich Ludwig Köchel. Ihr Wirken diente bereits der Quellenerschließung, einem Arbeitsvorgang, der gleichermaßen die charakteristischen Projekte der Musikgeschichtsforschung im 19. Jahrhundert, die Denkmäler der Tonkunst, sodann Biographien und auch die ersten Musikgeschichtsdarstellungen der neueren Zeit hervorbringt.

\section{POVZETEK}

Raziskovanje glasbene preteklosti se kot posebna stroka ukvarja s celotnim kompleksom kontekstov in tako glasbeno zgodovinopisje ne vprašuje le po dejstvih ampak tudi po smislu in metodah muzikalne historiografije. Zato naj bi bila teorija glasbene zgodovine bolj $v$ obzorju internacionalne muzikologije. Eno njenih izhodišč je zgodovina glasbenega zgodovinopisja. V smislu znanstvene discipline se to začenja šele konec 18. in na začetku 19. stoletja, kot razmišljanje o glasbenih stvareh pa se kažejo njegovi začetki že $v$ najstarejših mitih, $v$ staroorientalski genealogiji ter $\mathrm{v}$ spisih poznega helenizma in starih cerkvenih očetov.

Do obdobja racionalizma in prosvetljenstva je glasbeno zgodovinsko mišljenje večinoma apologetskega značaja in je vključeno $v$ religiozna in socialna vprašanja. $V$ kronističnih in enciklopedičnih spisih racionalizma in prosvetljenstva pa se začno postavljati glasbenozgodovinska vprašanja $v$ znanstvenem smislu. $K$ temu je bistveno prispevalo Herderjevo zgodovinsko mišljenje. Tako so nazadnje prav porajajoče se meščanske ideje o oblikovanju narodov in glasbeni omiki ljudstva kakor tudi predstave o obnavljanju in oživljanju stare glasbe pripeljale na začetku 19. stoletja do nastanka univerzitetnih kateder za glasbeno zgodovino in do razvoja glasbene zgodovine kot znanstvene discipline. 